\title{
SKYRMIONS AND THE $\nu=1$ QUANTUM HALL FERROMAGNET
}

\author{
M.J. Manfra, B.B. Goldberg \\ Dept. of Physics, Boston University, Boston MA 02215, USA \\ L. Pfeiffer and K. West \\ Bell Laboratories, Lucent Technologies, Murray Hill, NJ 07974, USA
}

\begin{abstract}
Recent experimental and theoretical investigations have resulted in a shift in our understanding of the $\nu=1$ quantum Hall state. There now exists a wealth of evidence that the excitation gap and the resulting quasiparticle spectrum at $\nu=1$ are due predominately to the ferromagnetic many-body exchange interaction. A great variety of experimentally observed correlations at $\nu=1$ cannot be incorporated into a perturbative expansion around the single-particle model, a scheme long thought to describe the integral quantum Hall effect at filling factor 1. Theorists now refer to the $\nu=1$ state as the quantum Hall ferromagnet. In this paper we review recent theoretical and experimental progress and detail our own optical investigations of the $\nu=1$ quantum Hall regime. The technique of magneto-absorption spectroscopy has proven to be powerful a probe of the occupancy of the lowest Landau level in the regime of $0.7<\nu<1.3$ about the spin gap. Additionally, we have performed simultaneous measurements of the absorption, photoluminescence and photoluminescence excitation spectra of the $\nu=1$ state in order to elucidate the role of excitonic and relaxation effects in optical spectroscopy in the quantum Hall regime.
\end{abstract}

PACS numbers: 73.40.Hm, 78.66.-w, 73.20.Mf, 71.70.Gm, 73.20.Dx

\section{Introduction}

Some fifteen years after the discovery of the fractional quantum Hall effect [1], the study of two-dimensional electron systems (2DES) confined to the lowest Landau level (LLL) continues to be a fertile laboratory for the investigation of many-body interactions. The proliferation of experimentally observed fractional Hall states [2], the discovery of composite fermions at half-filling [3], and the possibility for exotic spin-unpolarized fractional ground states [4] are but a few examples of the anomalies that continue to challenge our understanding of strong electron-electron correlations in the extreme magnetic quantum limit. Recently the $\nu=1$ quantum Hall state, a region thought to be well-understood within the 
single-particle model of the integral quantum IIall effect (IQHE), has been added to catalog of states whose physics is dominated by many-body interactions.

The importance of the many-body exchange coupling for determining the spectrum at $\nu=1$ can be seen in a fairly intuitive manner by looking at the relevant energy scales. The application of a large perpendicular magnetic field discretizes the single particle states of free electrons in two dimensions into Landau levels separated in energy by the cyclotron gap. Each Landau level is massively degenerate, allowing many electrons to have the same orbital energy. Each orbital Landau level is further split into a spin-up and spin-down state by the Zeeman coupling of the electron's spin to the external magnetic field. Filling factor $\nu=1$ corresponds to having exactly one electron for each available state in the lowest energy spin-up Landau level. Given the large magnetic fields typically necessary to observe the $\nu=1$ quantum Hall state ( $5 \mathrm{~T}<B<10 \mathrm{~T}$ ), it may at first appear odd to call this state the quantum Hall ferromagnet. Ferromagnetism is usually associated with the spontaneous alignment of spins in the absence of an external field. But at $\nu=1$ all the electrons share a common quantized kinetic energy which is conventionally chosen as the zero of energy. The gap to the next orbital Landau level is typically on the order of $200 \mathrm{~K}$ in units of temperature. Thus at low temperatures the electron's kinetic energy is essentially frozen out and does not affect the system's dynamics. The two remaining energy scales are the single-particle Zeeman energy and many-body exchange. In GaAs systems the Zeeman splitting is only of order $2 \mathrm{~K}$ while the typical electron-electron interaction scale is about $100 \mathrm{~K}$. A single spin reversal, which is the lowest energy single-particle excitation, costs a great deal in ferromagnetic exchange energy which is lowered by keeping neighboring spins aligned. Thus it may be that the physics of electrons interacting within a single orbital Landau level with such a small Zeeman coupling may not be appreciably different from that of a ferromagnet in the presence of a small symmetry breaking field. This is the essence of most recent theoretical approaches to the quantum Hall effect at $\nu=1$.

The notion of the quantum Hall ferromagnet was first advanced in the seminal work of Sondhi et al. [5]. His approach incorporated the use of the nonlinear $\sigma$ model (NL $\sigma$ ) of isotropic ferromagnets into the study of the $\nu=1$ quantum IIall effect. The NL $\sigma$ model has been extensively studied and its elementary excitations are known to possess highly nontrivial long-range spin order. These quasiparticles are called skyrmions and consist of a radial spin density that is reversed at the center but gradually heals to the ferromagnetic background over many magnetic lengths. Of course, real quantum Hall ferromagnets possess a small, but finite, Zeeman coupling which must be included for realistic calculations of energetics and spin polarization. An extended Hartree-Fock theory of quantum Hall skyrmions was developed by Fertig and coworkers [6] and provides a quantitative basis for comparison with experiment. The energetics and spin of real quantum Hall skyrmions, or charged spin texture excitations (CSTE's), are largely determined by the parameter $\tilde{g}=g \mu_{\mathrm{B}} B /\left(e^{2} / \epsilon_{0} l_{0}\right)$, the ratio of the Zeeman energy to the Coulomb interaction scale. The Zeeman term favors small skyrmions while the Coulomb term tries to maximize the size of the spin texture. For typical samples under investigation $\tilde{g} \approx 0.015$. For this value of $\tilde{g}$ the CSTE is always lower in 
energy than the single spin flip excitation and involves the reversal of approximately 3 spins. It is the excitation of skyrmions that is responsible for the rapid suppression of the spin polarization for excursions away from $\nu=1$.

\subsection{Experimental verification of skyrmions}

There now exists an extensive list of experimental results [7-12] that point unambiguously to existence of skyrmions as the lowest-lying charged excitation of the spin-polarized $\nu=1$ quantum Hall state. The initial data measuring the spin polarization as a function of filling factor came from Barrett's optically-pumped NMR studies [7]. His measurement of the Knight shift, which is proportional to the degree of electron spin polarization, shows a rapid and quasi-symmetric decay of the spin polarization for excursions away from $\nu=1$. His data indicated that each fundamental excitation consists of approximately 3 spin flips and could not be explained within the context of any single-particle model. A similar depolarization was evident in the magneto-absorption data of Aifer et al. [9]. Evidence for skyrmions has also been found in transport. Schmeller et al. [8] employed a tilted-field magneto-transport measurement of the energy gap at $\nu=1$. By tilting the sample in a magnetic field while keeping the filling factor constant the ratio of the Zeeman energy to the Coulomb energy can be varied. Schmeller measured the activation gap as a function of tilting angle. The activation energy is sensitive to the long-wavelength charged excitations of the system and its variation implied charge transport mediated by quasiparticles carrying large spin quantum numbers. All of these early measurements pointed to the dominant role played by ferromagnetic exchange and were in good agreement with theory that incorporated skyrmions as the fundamental excitation of the system.

\section{Optical detection of skyrmions}

Our determination of the spin polarization of the $\nu=1$ state is based on polarization-resolved magneto-absorption spectroscopy. The 2DES resides at the interface of a single-side $n$-modulation doped $\mathrm{AlGaAs}$-GaAs single quantum well (SQW). The well thickness is $250 \AA$ with an electron density of $N_{\mathrm{s}}=1.8 \times 10^{11} \mathrm{~cm}^{-2}$ and mobility $\mu=2.6 \times 10^{6} \mathrm{~cm}^{2} /(\mathrm{V} \mathrm{s})$. In order to perform absorption measurements the samples were mounted strain-free and thinned to $\approx 0.5 \mu \mathrm{m}$ with a jet-etching technique. Collimated light centered at $800 \mathrm{~nm}$ is passed to, and collected from, the sample through a fiber optic system that fits in a $\mathrm{He}^{3}$ refrigerator mounted in a $12 \mathrm{~T}$ magnet. Polarization analysis is done in situ with a circular analyzer placed immediately following the sample. Reversing the direction of the magnetic field relative to the direction of propagation of light allows for the independent monitoring of the left circularly polarized (LCP) and right circularly polarized (RCP) spectra.

Magneto-absorption spectroscopy has several advantages that make it a unique probe of the spin polarization of the 2DES. Firstly, the integrated absorption intensity is directly proportional to the available density of states in the lowest Landau level and not dependent on any complicated relaxation effects. Additionally, the angular momentum states of the initial and final states are well-defined 


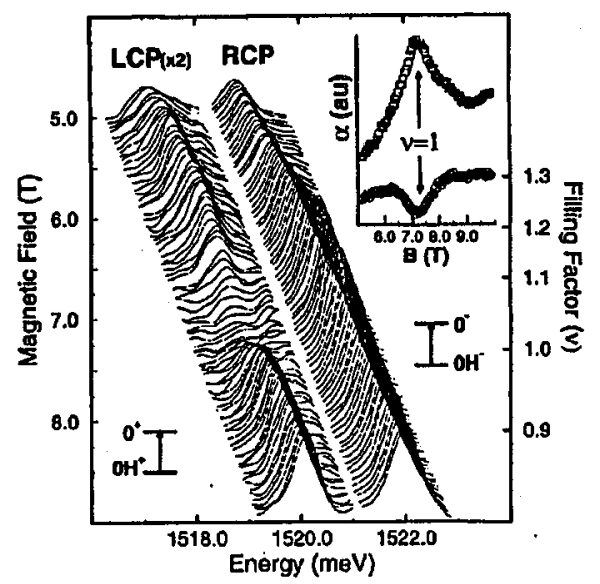

Fig. 1. Absorption spectra in LCP and RCP as a function of magnetic field at $T=$ 1.5 K. The RCP spectra are offset by $+1 \mathrm{meV}$ in energy for clarity. The main optical transitions to the two lowest electron spin states are shown alongside the spectra. The inset displays the peak absorption coefficient $\alpha$ in the vicinity of $\nu=1$.

so that by monitoring both the LCP transition and the RCP transition we can independently monitor the populations of the two spin bands of the lowest Landau level as the Fermi level sweeps through $\nu=1$.

An example of the absorption spectra as a function of magnetic field in each polarization is shown in Fig. 1. As the Fermi level moves through $\nu=1$ the absorption to the lower energy spin-up state quenches, concomitant with a peaking of the absorption into the higher energy spin-down state. This is clearly seen in the inset to Fig. 1 where the absorption coefficient $\alpha=-1 / L_{\mathrm{w}} \ln (I(B) / I(0))$ ( $L_{\mathrm{w}}$ is the quantum well width and $I$ the measured transmission intensity) is plotted as a function of $B$ in the neighborhood of $\nu=1$ for each polarization. These data indicate the rapidly changing populations of the two spin states on both the high and low field sides of $\nu=1$.

Our determination of the spin polarization from the absorption spectra follows from Fermi's golden rule, which relates the integrated absorption intensity to the available density of states in each spin band, and on a simple sum rule which enforces particle conservation,

$$
\frac{N_{\uparrow}+N_{\downarrow}}{N}=1
$$

where $N_{\uparrow(\downarrow)}$ is the number of spin-up (down) electrons in the lowest Landau level. This can be recast as

$$
\frac{N_{\mathrm{A}_{1}}+N_{\mathrm{A}_{\mathrm{t}}}}{N}=\frac{2-\nu}{\nu},
$$

where $N_{\mathrm{A}_{\mathrm{T}(1)}}$ is the available density of states in the spin-up (down) band of the lowest Landau level. The sum rule constrains the total available density of states at any given magnetic field. We proceed with the calculation of $S_{z}$ by first dividing the 
integrated peak absorption in each polarization by the calculated optical matrix elements. The resulting quantity is proportional to the available density of states

$$
\frac{I_{i j}}{f_{i j}}=C N_{\mathrm{A}_{j}},
$$

where $I_{i j}$ is the integrated absorption, $f_{i j}$ is the optical matrix element and $C$ is the constant of proportionality to be determined. Since the left and right circularly polarized spectra provide two such independent equations - one for each spin band - the additional constraint enforced by (2) allows for the elimination of the constant of proportionality $C$ and the determination of $N_{\mathrm{A}_{\mathrm{t}(1)}}$. Finally the spin polarization per particle is

$$
S_{z}=\frac{N_{\uparrow}-N_{\downarrow}}{N}=\frac{N_{\mathrm{A}_{\downarrow}}-N_{\mathrm{A}_{\uparrow}}}{N} \text {. }
$$

Note that the final expression for $S_{z}$ is completely independent of $C$, the unknown constant of proportionality. Thus this experiment allows for the determination of the absolute spin polarization, free of any fitting parameters. The constant of proportionality $C$ systematically accounts for all unmeasured experimental couplings - optical collection efficiency, detector efficiency, etc. - which otherwise would be needed to relate the measured integrated intensity to the available density of states. Furthermore, the sum rule which determines the proportionality between the measured absorption and the density of final states can be made rigorous when a full integration over energy is used. The energy integrated absorption is independent of excitonic or many-body interactions and can be understood simply as counting the total number of a vailable $k$-states in the system [13]. The uncertainty is in determining the cut-off energy for the integration. Since the optical transitions are well separated in energy, it suffices to use the transitions which have as final states the spin-up and spin-down states of the lowest Landau level.

Figure 2 plots the spin polarization versus filling factor determined from the data in Fig. 1 and compares it with both single-particle and skyrmion-based models. Previous calculations have shown that a single-particle model based on the exchange enhanced $g$-factor [14] that modulates the overlap of the two electron spin levels fails to capture the behavior of $S_{z}$, especially for $\nu<1$ [9]. On the other hand, the data conform well to the skyrmion-based model. In this scheme the number of reversed spins is quantized [15] so that the component of total spin along the direction of the Zeeman field is given by

$$
S_{z}=N / 2-(A+1)\left|N-N_{\phi}\right|
$$

for $N>N_{\phi}$, where $N$ is the number of electrons in the system, $N_{\phi}$ is the number of states available and $\left|N-N_{\phi}\right|$ is the number of skyrmions present. For $N<N_{\phi}, S_{z}$ is given by

$$
S_{z}=N / 2-(A)\left|N-N_{\phi}\right| \text {. }
$$

$A$ is an integer quantum number which depends on the relative strength of the Zeeman and Coulomb interaction terms. It is the relevant measure of the skyrmion size [15]. Hartree-Fock calculations [6] predict that for quantum Hall ferromagnets with our material parameters $A$ should be close to 3 , indicating each excitation induces 3 spin flips. Our data are in excellent agreement with this prediction. Note 


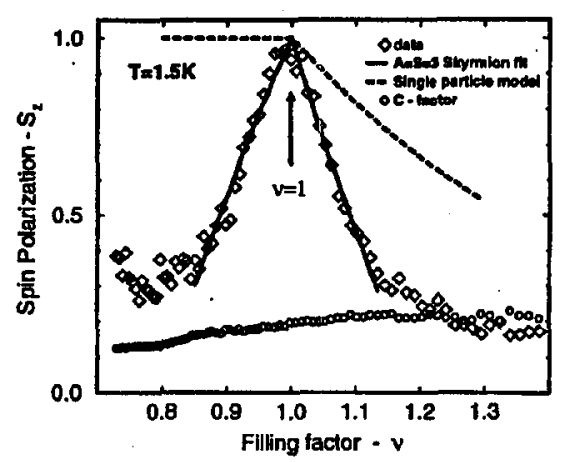

Fig. 2. Spin polarization plotted versus filling factor and compared with both a single particle and skyrmion-based model. The single particle polarization is based on a simple counting argument, one spin flip per unpaired flux quantum for $\nu>1$ and $S_{z}=1$ for $\nu<1$. The skyrmion model has been detailed elsewhere [10] and fits well for 3 spin flips per unpaired flux quanta. The magnetic field dependence of the constant of proportionality $C$ is also shown.

that the measured spin polarization does indeed approach unity exactly at $\nu=1$ as $T \rightarrow 0$, indicating that the ground state at $\nu=1$ is fully spin polarized. Finally, the constant of proportionally $C$ is also displayed in Fig. 2. The complete lack of structure observed in $C$ over a field range in which the spin polarization changes from nearly zero to unity is good indication that our simple sum rule is obeyed.

\subsection{Temperature dependence of spin polarization at $\nu=1$}

The discussion so far has focused on the skyrmionic excitations of the quantum Hall ferromagnet. Skyrmions are the charged excitations of the system and are driven by adding or removing charge, or equivalently, adding or removing magnetic flux. The charged nature of skyrmions is evidenced by the sensitivity of transport measurements to their presence $[8,12]$. But skyrmions are not the only excitations of the quantum Hall ferromagnet. As with any highly correlated spin system, we expect a branch of low-lying neutral spin-wave excitations which may affect the spin polarization even at $\nu=1$ for finite temperatures. In order to explore the nature of the neutral excitations of the quantum Hall ferromagnet we have completed a detailed study of the thermal behavior of the spin polarization exactly at $\nu=1$.

Band-gap absorption spectra show striking temperature dependence due to changes in the occupations of the spin-split states of the ground Landau level at filling factors near $\nu=1$. Knowledge of the temperature dependent occupations of the spin-up $\left(0^{+}\right)$and spin-down ( $\left.0^{-}\right)$electron levels leads to a thermodynamic measure of the spin polarization. Figure 3 displays the temperature dependence of the absorption taken in LCP and RCP at $\nu=1$. The decrease in absorption for decreasing temperature into the lower energy spin state in LCP is correlated to the increase in absorption into the higher energy spin state in RCP. As the temperature decreases, there are fewer available states for optical transitions in the 


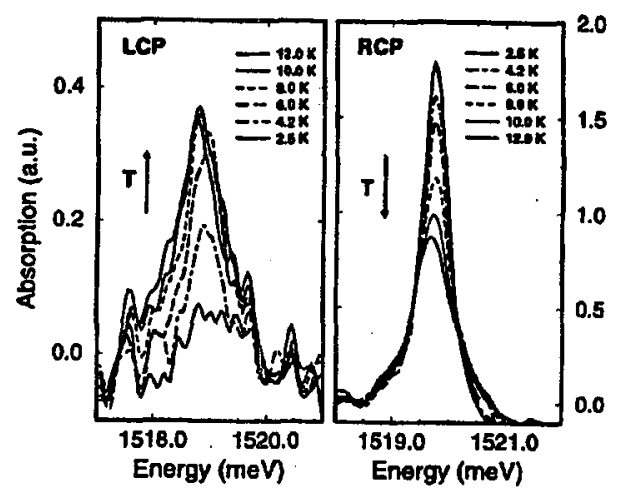

Fig. 3. Absorption spectra taken in LCP and RCP at $\nu=1$ as a function of temperature. The arrows point in the direction of spectral change for increasing temperature.

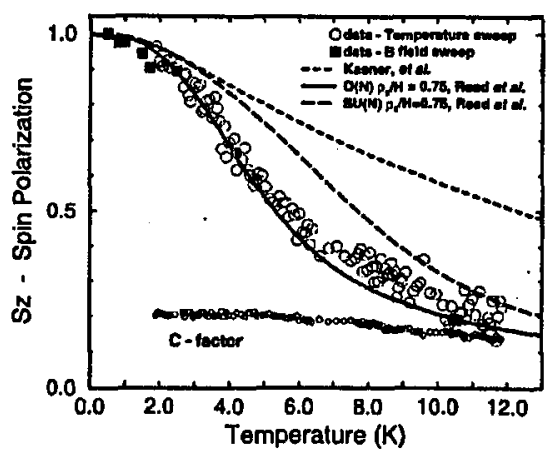

Fig. 4. Temperature dependence of the spin polarization at $\nu=1$ as determined by polarized absorption spectroscopy. The theoretical curves from the CQFM of Read et al. and the many-body perturbation curve of Kasner et al. are also displayed.

lower-energy spin component $\left(0^{+}\right)$and more available in the higher-energy spin component $\left(0^{-}\right)$. This data is convincing evidence that the temperature-dependent absorption monitors the Fermi distribution of a two-level system. $S_{z}$ vs. $T$ is plotted from $500 \mathrm{mK}$ to $12 \mathrm{~K}$ in Fig. 4 . It is important to note that $C$ is independent of temperature (see Fig. 4), giving further indication that only the occupancy of the two levels is changing over this temperature range. Additionally we display several values of $S_{z}(T)$ obtained independently by sweeping magnetic field at constant temperature. The consistency between the spin polarization determined from magnetic field sweeps and the spin polarization determined from temperature sweeps is very good.

The rapid quenching of the $\nu=1$ polarization with increasing temperature is further evidence for the highly correlated nature of the quantum Hall ferromagnet. Any theoretical approach to its thermodynamics must account for the collective-magnetization excitations of the ferromagnetic ground state which are expected to dominate the finite-temperature spin polarization. Initial theoretical 
work [16] which included independent spin-waves displays a much weaker temperature dependence of the spin polarization than our data indicate. Kasner and MacDonald have incorporated spin-wave excitations into a many-body perturbation theory through the inclusion of a self-energy insertion consisting of a ladder sum of repeated interactions between Hartree-Fock (HF) electrons of one spin and holes of the opposite spin [17]. Their theoretical $S_{z}$ vs. $T$ curve appropriate to our experimental conditions, including finite well thickness effects, is shown in Fig. 4. The low-temperature reduction of $S_{z}(T)$ is dominated by the long-wavelength spin-wave contribution and compares favorably with the data. At higher temperatures however, agreement is less convincing. This perturbative approach is limited by its inability to consistently account for spin-wave-spin-wave interactions.

Read and Sachdev have developed a continuum quantum field theory of systems with a ferromagnetic ground state that is applicable to the $\nu=1$ quantum Hall state [18]. The description is analogous to an insulating quantum Heisenberg ferromagnet, and the low-temperature behavior of the two systems is expected to be similar. The continuum quantum ferromagnet (CQFM) contains a conserved topological current representing the number density and current of skyrmions. Most importantly to our discussion of the $\nu=1$ spin thermodynamics, the finite temperature CQFM systematically accounts for spin-wave-spin-wave interactions which dominate the spin thermodynamics in the regime $k_{\mathrm{B}} T>H$, where $H=g \mu_{\mathrm{B}} B$ is the Zeeman energy. The only parameter in the system is set by the ratio of the energy scales $\rho_{\mathrm{s}}$, the ferromagnetic spin stiffness, and $H$. In the limit of zero well thickness, $\rho_{\mathrm{s}}=e^{2} /\left(16 \sqrt{2 \pi} \epsilon l_{\mathrm{B}}\right)$. The ratio calculated for our GaAs SQW, including the effects of finite well thickness, is $\rho_{\mathrm{s}} / H \approx 0.77$ [19]. Scaling functions for the spin polarization can be generated in the large $N$ limit when the symmetry group $\mathrm{O}(3)$ is generalized to $\mathrm{O}(N)$ or $\mathrm{SU}(N)$. The large $N$ expansion is not a perturbative expansion in the strength of the interactions but rather a saddle point expansion which preserves the symmetry and couplings of the underlying Hamiltonian. Thus evaluating the spin polarization for both $\mathrm{O}(N)$ and $\mathrm{SU}(N)$ in the $N \rightarrow \infty$ limit does not correspond to choosing different symmetry groups for the physical system since $\mathrm{O}(3) \cong \mathrm{SU}(2)$. It will, however, alter the resulting form of the scaling functions at the mean field level ${ }^{*}$. In Fig. 4 the $\mathrm{O}(N)$ and $\mathrm{SU}(N)$ limits of the CQFM with $\rho_{\mathrm{s}} / H=0.75$ are displayed. We find excellent agreement over the entire range of measured temperatures. It is important to note that there are no free parameters in this fit to the data. All couplings are known exactly and are specific to our system. It is clear that the physics of collective-magnetization excitations captured by the CQFM is crucial to reproducing the observed temperature dependence of $S_{z}$.

\section{2. $P L$ and PLE spectroscopy at $\nu=1$}

It is apparent that magneto-absorption spectroscopy is a sensitive probe of the occupancy of the spin-split lowest Landau level. Yet its application to spectroscopy of the quantum Hall regime remains limited to a few studies $[9,11,20,21]$.

*The extent of the systematic errors introduced in going to the large $N$ limit are unclear. Any difference between $\operatorname{SU}(N)$ and $\mathrm{O}(N)$, which are the same theory if $N=2$ and $N=3$ respectively, may suggest the level of theoretical uncertainty. $N$. Read, private communication. 
A decade's worth of published photoluminescence (PL) data [22] exists with no clear PL signature of skyrmions yet found. In order to clarify the differences between the various optical probes we have recently undertaken the simultaneous measurement of the absorption, PL and PLE spectra from the quantum Hall ferromagnet. Though experimentally more cumbersome, spectra obtained simultaneously allow for much more meaningful comparisons. Ilere differences due to sample preparation, multiple cool downs, changes in optical coupling and different field and temperature sweeps may be avoided.

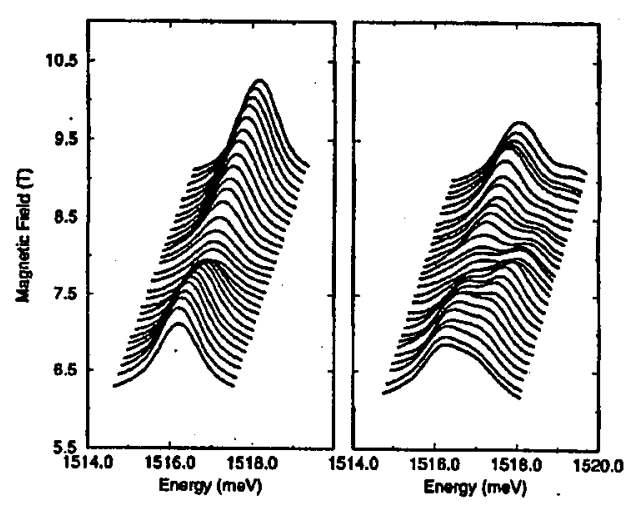

Fig. 5. Photoluminescence spectra taken in LCP and RCP at $\nu=1$ as a function of magnetic field $(T=0.5 \mathrm{~K})$.

Figure 5 displays low temperature photoluminescence spectra in both LCP and RCP polarizations in the region of $\nu=1$ arising from the recombination of electrons in the two spin states of the lowest Landau level with valence band holes. In comparison to the absorption spectra (see Fig. 1) the PL spectra exhibit much more complicated behavior where the interplay of excitonic final state interactions, QHE localization, and many-body screening effects are known to alter the ground state recombination. We begin consideration of the emission process with the LCP polarization. Its energy position and polarization identify this emission as ground state recombination resulting from the transition $0^{+} \rightarrow \mathrm{OH}^{+}$. A clear quenching of intensity is visible as the magnetic field is swept through $\nu=1$. This quenching has a monotonic temperature dependence between $4.2 \mathrm{~K}$ and $1.5 \mathrm{~K}$ but interestingly saturates for temperatures below $1.5 \mathrm{~K}$. Additionally the emission asymmetrically broadens to the low energy side at $\nu=1$, increasing its line width by a factor of 2 . This observation is consistent with recent theoretical considerations which predict an asymmetric low-energy broadening due to low energy spin-waves left behind in the final state upon the emission of LCP photons [23].

Even more complicated structure is visible in the RCP polarization where two distinct peaks are clearly visible in the field range of $6.7 \mathrm{~T}$ to $8.2 \mathrm{~T}$. We assign the high energy peak (HE) to the recombination of electrons in the spin-down state of the LLL with valence band holes $\left(0^{-} \rightarrow \mathrm{OH}^{-}\right)$. This is the inverse of the absorption process seen in $\mathrm{RCP}\left(\mathrm{OH}^{-} \rightarrow 0^{-}\right)$and is only seen in the RCP 
polarization. This observation is consistent with our hole level calculations which show no mixing of the $\mathrm{OH}^{-}$state, indicating a very clean transition. There is a clear peaking of the high energy emission as one approaches $\nu=1$ from the low field side and a very rapid loss of HE intensity as one moves to lower filling factors. A lower energy peak (LE) is also visible in RCP which, due to the rather broad line widths of the emission process $(\approx 1.5 \mathrm{meV})$, overlaps with the HE emission. This emission is attributed to the recombination of ground state spin-up electrons through an allowed RCP transition. The fact. that this is ground state emission through an allowed RCP channel is supported by its peak position as a function of magnetic field. Within our experimental resolution this peak coincides in energy with the main ground state emission seen in LCP. This is one of the most glaring differences we see between the absorption and PL processes - while the absorption spectra are extremely clean with respect to polarization selection rules, the emission is much more sensitive to angular momentum mixing of the photo-excited hole states. Additionally the absorption spectra exhibit none of the changes in line widths as the Fermi level is swept through $\nu=1$ that are seen in the luminescence. Clearly the hole relaxation processes are crucial to understanding the emission while absorption is not so sensitive to these effects.

Given the seemingly different behaviors observed in PL and absorption, we have measured the photoluminescence excitation (PLE) spectra in order to investigate possible relaxation mechanisms. Figure 6 displays the response of the ground state emission in LCP at $\nu=1$ and $T=0.5 \mathrm{~K}$. Also displayed in this figure are the LCP and RCP absorption coefficients taken at the same time. The correspondence between the luminescence and the combined absorption coefficient is striking. The energy positions coincide to within experimental resolution and the relative change in emission intensity maps exactly onto the relative changes in the absorption coefficient. This is very telling data. The ground state emission in LCP

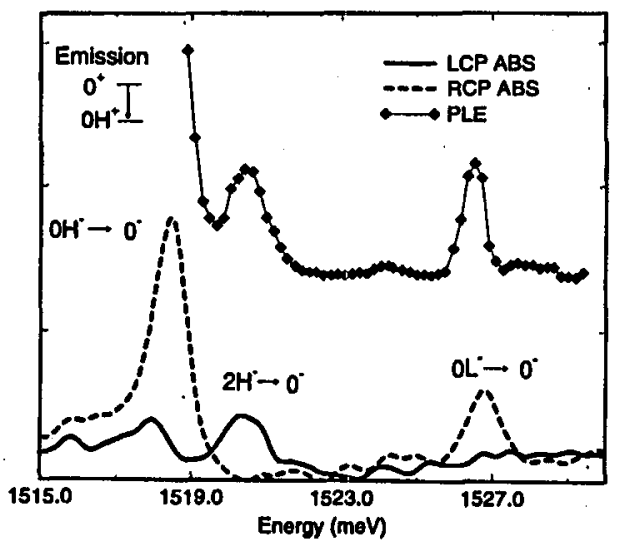

Fig. 6. Integrated ground state emission intensity at $\nu=1$ as a function of laser excitation energy. Also displayed are the LCP and RCP direct absorption versus energy at $\nu=1$. Main absorption transitions are indicated $(T=0.5 \mathrm{~K})$. 
is tracking both the LCP and RCP absorption. An indication of such mixing was seen in the lack of strict polarization in the non-resonant ground state emission. The PLE spectra further point to the importance of the hole relaxation in the luminescence process. The emission in LCP increases whenever we have absorption, regardless of whether we are creating LCP or RCP holes. Thus holes created at absorption must change angular momentum character before recombining.

As the data indicate, the interpretation of luminescence is nontrivial and dependent upon a detailed knowledge of final state interactions. Extracting the relative spin populations of the lowest Landau is much more readily accomplished through magneto-absorption spectroscopy. A detailed study of PLE spectra in the quantum Hall regime is presently under investigation and the subject of future publication. Additionally, several recent theoretical efforts have focused on finding a unambiguous PL signature of skyrmions [24, 23]. A detailed comparison of our PL data with theory is presently underway.

\section{Acknowledgments}

This work was supported by National Science Foundation grant No. DMR-9158097. We are thankful for helpful and illuminating discussions with N. Read, S. Sachdev, A. MacDonald, M. Kasner and N. Cooper. Finite well thickness corrections to the 2D spin stiffness were kindly provided by A. MacDonald and M. Kasner.

\section{References}

[1] D.C. Tsui, H.L. Stormer, A.C. Gossard, Phys. Rev. Lett. 48, 1559 (1982).

[2] R.L. Willet, J.P. Eisenstein, H.L. Stormer, D.C. Tsui, A.C. Gossard, J.H. English, Phys. Rev. Lett. 59, 1776 (1987).

[3] H.W. Jiang, H.L. Stormer, D.C. Tsui, L.N. Pfeiffer, K.W. West, Phys. Rev. B 40, 12013 (1989).

[4] T. Chakraborty, P. Pietilainen, The Fractional Quantum Hall Effect, Springer-Verlag, New York 1988.

[5] S.L. Sondhi, A. Karlhede, S.A. Kivelson, E.II. Rezayi, Phys. Rev. B 47, 16419 (1993) and references therein.

[6] H.A. Fertig, L. Brey, R. Cote, A.H. MacDonald, Phys. Rev. B 50, 11018 (1994).

[7] S.E. Barrett, G. Dabbagh, L.N. Pfeiffer, K.W. West, Z. Tycko, Phys. Rev. Lett. 74, 5112 (1995).

[8] A. Schmeller, J.P. Eisenstein, L.N. Pfeifer, K.W. West, Phys. Rev. Lett. 75, 4290 (1995).

[9] E.H. Aifer, B.B. Goldberg, D.A. Broido, Phys. Rev. Lett. 76, 680 (1996).

[10] V. Bayot, E. Grivei, S. Melinte, M.B. Santos, M. Shayegan, Phys. Rev. Lett. 76, 4584 (1996).

[11] M.J. Manfra, E.H. Aifer, B.B. Goldberg, D.A. Broido; L. Pfeiffer, K. West, Phys. Rev. B Rapid Commun. 54, 17327 (1996).

[12] D.K. Maude, M. Potemski, J.C. Portal, M. Henini, L. Eaves, G. Hill, M.A. Pate, Phys. Rev. Lett. 77, 4604 (1996).

[13] N.R. Cooper, private communication. 
[14] T. Ando, Y. Uemura, J. Phys. Soc. Japan 37, 1044 (1974).

[15] A.H. MacDonald, H.A. Fertig, L. Brey, Phys. Rev. Lett. 76, 2153 (1996).

[16] C. Kallin, B.I. Halperin, Phys. Rev. B. 30, 5655 (1984).

[17] M. Kasner, A.H. MacDonald, Phys. Rev. Lett. 76, 3204 (1996).

[18] N. Read, S. Sachdev, Phys. Rev. Lett. 75, 3509 (1995).

[19] A.H. MacDonald, M. Kasner, private communication.

[20] B.B. Goldberg, D. Heiman, A. Pinczuk, Phys. Rev. Lett. 63, 1102 (1989).

[21] B.B. Goldberg, D. Heiman, M.J. Graf, D.A. Broido, A. Pinczuk, C.W. Tu, J.H. English, A.C. Gossard, Phys. Rev. B 38, 10131 (1988).

[22] B.B. Goldberg, D. Heiman, A. Pinczuk, L. Pfeiffer, K. West, Phys. Rev. Lett. 65, 641 (1990); A.J. Turberfield, S.R. Haynes, P.A. Wright, R.A. Ford, R.G. Clark, J.F. Ryan, J.J. Harris, C.T. Foxon, Phys. Rev. Lett. 65, 637 (1990); B.B. Goldberg, D. Heiman, M. Dahl, A. Pinczuk, L. Pfeiffer, K. West, Phys. Rev. B 44, 4006 (1991); E.M. Goldys, S.A. Brown, R.B. Dunford, A.G. Davies, R. Newbury, R.G. Clark, P.E. Simmonds, J.J. Harris, C.T. Foxon, Phys. Rev. B 46, 7957 (1992); M. Dahl, D. Heiman, A. Pinczuk, B.B. Goldberg, L.N. Pfeiffer, K. West, Phys. Rev. B 45, 6957 (1992).

[23] N.R. Cooper, D.B. Chklovskii, Phys. Rev. B 55, 2436 (1997).

[24] T. Portengen, J.R. Chapman, V.N. Nicopoulos, N.F. Johnson, Phys. Rev. B 55, 7367 (1997). 